\title{
Analytical solution of Abel integral equation
}

\author{
E. A. A. Ziada \\ Nile Higher Institute for Engineering \& Technology, Mansoura, Egypt. \\ eng_emanziada@yahoo.com
}

\begin{abstract}
In this paper, we apply the Adomian decomposition method (ADM) to solve Abel integral equation of the first and second kind. Abel integral equation is one the most important equations which appear in a lot of applications.
\end{abstract}

Keywords: Adomian Method; Abel integral equation; fractional integral equation.

\section{Introduction}

In this paper, we shall consider one of the most important fractional integral equations, namely the Abel integral equations of the first and the second kind;. The former investigations on such equations are due to Abel (1823), after whom they are named, for the first kind, and to Hille and Tamarkin (1930) for the second kind. The interested reader is referred to [1] and [2]-[6] for historical notes and detailed analysis with applications. Some of these applications are, in evaluation of spectroscopic measurements of cylindrical gas discharges, the study of the solar or a planetary atmosphere, the investigation of star densities in a globular cluster, the inversion of travel times of seismic waves for determination of terrestrial sub-surface structure, the tautochrone problem, spherical stereology. We use Adomian decomposition method (ADM) [5][10] to solve these kind of equations. This method has many advantages; it is efficiently works with different types of linear and nonlinear equations in deterministic or stochastic fields and gives an analytic solution for all these types of equations without linearization or discretization. 


\section{Problem solving}

\subsection{Abel integral equation of the first kind}

Let us consider the Abel integral equation of the first kind,

$$
\frac{1}{\Gamma(\alpha)} \int_{0}^{t} \frac{u(\tau)}{(t-\tau)^{1-\alpha}} d \tau=f(t), \quad 0<\alpha<1,
$$

where $f(t)$ is a given function. We easily recognize that this equation can be expressed in terms of a fractional integral as follow,

$$
J^{\alpha} u(t)=f(t),
$$

and consequently solved in terms of a fractional derivative according to,

$$
u(t)=D^{\alpha} f(t) .
$$

\subsection{Abel integral equation of the second kind}

Let us now consider the Abel equation of the second kind,

$$
u(t)+\frac{\lambda}{\Gamma(\alpha)} \int_{0}^{t} \frac{u(\tau)}{(t-\tau)^{1-\alpha}} d \tau=f(t), \quad \alpha>0,
$$

This equation can be expressed in terms of a fractional integral as,

$$
u(t)+\lambda J^{\alpha}[u(t)]=f(t),
$$

this problem was solved before in [14] by using Laplace transform method. This solution is,

$$
u(t)=f(t)+e_{\alpha}^{\prime}(t ; \lambda) * f(t)
$$

where,

$$
e_{\alpha}^{\prime}(t ; \lambda)=\frac{d}{d t} E_{\alpha}\left(-\lambda t^{\alpha}\right)=\infty n=1 \sum(-\lambda)^{n} \frac{t^{\alpha n-1}}{\Gamma(\alpha n)}, \quad t>0 .
$$

Now, we will solve it by using ADM. Equation (5) can be written as,

$$
u(t)=f(t)-\lambda J^{\alpha}[u(t)],
$$

Using ADM we have,

$$
\begin{gathered}
u_{0}(t)=f(t), \\
u_{n}(t)=-\lambda J^{\alpha}\left[u_{n-1}(t)\right], \quad n \geq 1 .
\end{gathered}
$$

\subsection{Application}

We are going to describe in detail the occurrence of Abel integral equations of first and of second kind in the problem of heating (or cooling) of a semi-infinite rod by influx (or efflux) of heat across the boundary into (or from) its interior.

Consider the equation of heat flow [14],

$$
u_{t}-u_{x x}=0, \quad u=u(x, t),
$$

in the semi-infinite intervals $0<x<\infty$ and $0<t<\infty$ of space and time, respectively. In this 
dimensionless equation $u=u(x, t)$ means temperature. Assume vanishing initial temperature, i.e. $u(x, 0)=0$ for $0<x<\infty$ and given influx across the boundary $x=0$,

$$
-u_{x}(0, t)=p(t) \text {. }
$$

Then, under appropriate regularity conditions, $u(x, t)$ is given by the formula, see e.g. [15],

$$
u(x, t)=\frac{1}{\sqrt{\pi}} \int_{0}^{t} \frac{p(\tau)}{\sqrt{t-\tau}} e^{-x^{2} /[4(t-\tau)]} d \tau, \quad x>0, t>0 .
$$

We turn our special interest to the interior boundary temperature $\phi(t)=u\left(0^{+}, t\right), t>0$, which by (12) is represented as,

$$
\frac{1}{\sqrt{\pi}} \int_{0}^{t} \frac{p(\tau)}{\sqrt{t-\tau}} d \tau=J^{1 / 2} p(t)=\phi(t), \quad t>0 .
$$

We recognize (3.128) as an Abel integral equation of first kind for determination of an unknown influx $p(t)$ if the interior boundary temperature $\phi(t)$ is given by measurements, or intended to be achieved by controlling the influx. Its solution is given by formula (3) as,

$$
p(t)=D^{1 / 2} \phi(t)
$$

By using equation (14), we can find the value of $p(t)$.

Let us take the following special cases,

$$
\begin{aligned}
& \text { (i) } \phi(t)=t \Rightarrow p(t)=\frac{1}{2} \sqrt{\pi t}, \\
& \text { (ii) } \phi(t)=1 \Rightarrow p(t)=\frac{1}{\sqrt{\pi t}} .
\end{aligned}
$$

We now modify our problem to obtain an Abel integral equation of second kind. Assume that the rod $x>0$ is bordered at $x=0$ by a bath of liquid in $x<0$ with controlled exterior boundary temperature $u\left(0^{-}, t\right)=\psi(t)$.

Assuming Newton's radiation law we have an influx of heat from $0^{-}$to $0^{+}$proportional to the difference of exterior and interior temperature,

Inserting (15) into (13), we obtain

$$
p(t)=\lambda[\psi(t)-\phi(t)], \quad \lambda>0 .
$$

$$
\phi(t)=\frac{\lambda}{\sqrt{\pi}} \int_{0}^{t} \frac{\psi(\tau)-\phi(\tau)}{\sqrt{t-\tau}} d \tau
$$

which can be written as,

$$
\phi(t)+\lambda J^{1 / 2}[\phi(t)]=\lambda J^{1 / 2}[\psi(t)] .
$$

If we now assume the exterior boundary temperature $\psi(t)$ as given and the evolution in time of the interior boundary temperature $\phi(t)$ as unknown, then (17) is an Abel integral equation of second kind for determination of $\phi(t)$.

The solution given in [14] of the problem (17) for $\psi(t)=1$, by using the Laplace transform method is,

$$
\phi(t)=1-E_{1 / 2}\left(-\lambda t^{1 / 2}\right)
$$

Using ADM to problem (17), the recursive relations will be, 


$$
\begin{gathered}
\phi_{0}(t)=\lambda J^{1 / 2}[\psi(t)]=\lambda J^{1 / 2}[1]=\lambda \frac{t^{1 / 2}}{\Gamma(3 / 2)}, \\
\phi_{n}(t)=-\lambda J^{1 / 2}\left[\phi_{n-1}(t)\right], \quad n \geq 1 .
\end{gathered}
$$

From relation (19), the first five-terms of the series solution will be,

$$
\phi(t)=\frac{2 \lambda \sqrt{t}}{\sqrt{\pi}}-t \lambda^{2}+\frac{4 t^{3 / 2} \lambda^{3}}{3 \sqrt{\pi}}-\frac{t^{2} \lambda^{4}}{2}+\frac{8 t^{5 / 2} \lambda^{5}}{15 \sqrt{\pi}}-\cdots .
$$

A comparison between ADM solution and Laplace solution for $\lambda=1$ is given in figures 1.a-1.c.

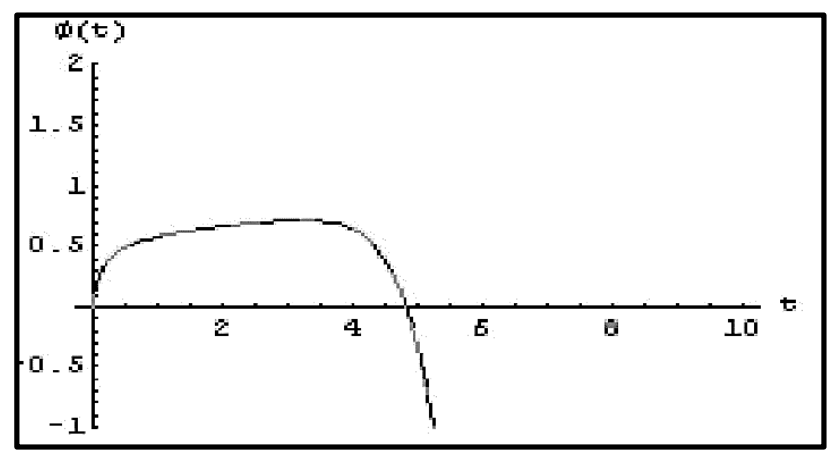

Fig. 1.a: ADM and Laplace Sol. $[n=20]$

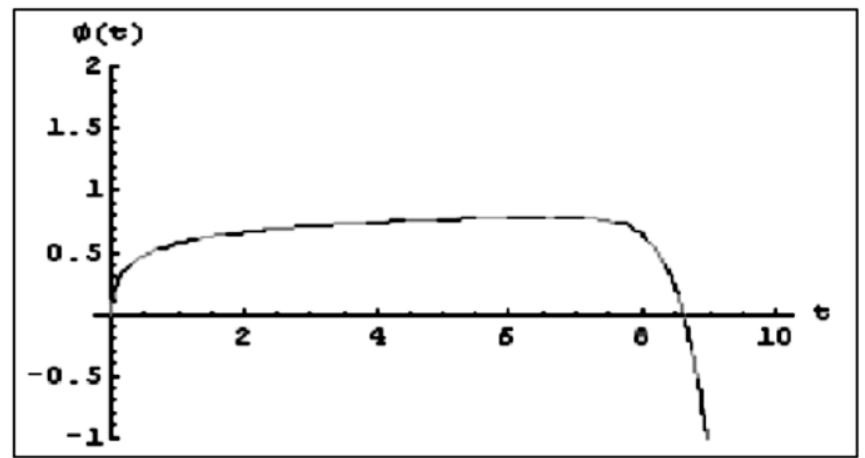

Fig. 1.b: ADM and Laplace Sol. $[n=40]$

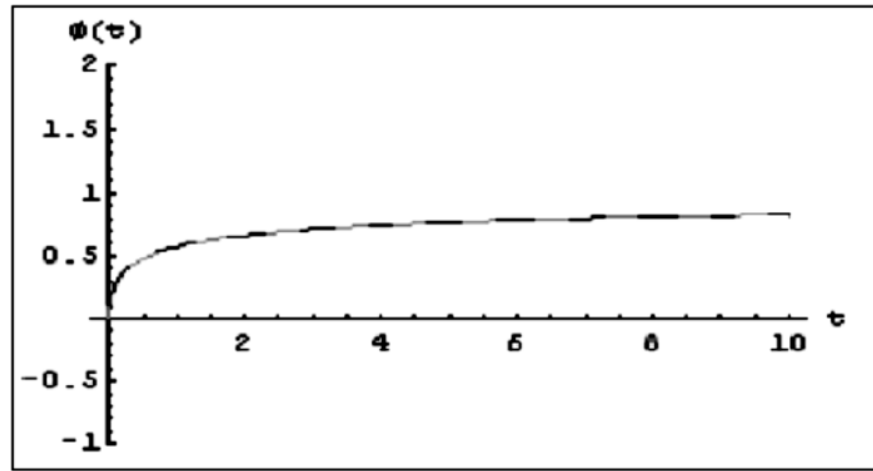

Fig. 1.c: ADM and Laplace Sol. $[n=60]$ 


\section{References}

[1] S. G. Samko, A. A. Kilbas and O. I. Maritchev, Fractional Integrals and Derivatives, Theory and Applications, Gordon and Breach, Amsterdam 1993.

[2] R. Gorenflo, and S. Vessella, Abel Integral Equations: Analysis and Applications, Lecture Notes in Mathematics \# 1461, Springer-Verlag, Berlin 1991.

[3] J. D. Craig, and J. C. Brown, Inverse Problems in Astronomy Adam Hilger Ltd, Bristol 1986.

[4] R. Gorenflo, Abel Integral Equations with special Emphasis on Applications, Lectures in Mathematical Sciences, 1996. (ISSN 0919-8180)

[5] R. Gorenflo, The tomato salad problem in spherical stereology, in Transform Methods and Special Functions, Varna 1996 (Eds. Rusev, P. Dimovski, I and V. Kiryakova), Science Culture Technology, Singapore 1997 (in press). [Proc. Int. Workshop, Varna, Bulgaria, 23-30 August 1996] An extended version is available as Pre-print A-25/96, Fachbereich Mathematik und Informatik, Freie Universität, Berlin 1996, via Internet: <http: //www.math.fu-berlin.de/publ/index.html>

[6] N. Wheeler, Construction \& Physical Application of the Fractional Calculus, Reed College Physics Department, 1997, pp. 1-59.

[7] G. Adomian, "Solving Frontier Problems of Physics: The Decomposition Method", Kluwer, (1995).

[8] G. Adomian, "Stochastic System", Academic press, (1983).

[9] G. Adomian, "Nonlinear Stochastic Operator Equations", Academic press, San Diego, (1986).

[10] G. Adomian, "Nonlinear Stochastic Systems: Theory and Applications to Physics", Kluwer, (1989).

[11] K. Abbaoui, and Y. Cherruault, "Convergence of Adomian's method Applied to Differential Equations", Computers Math. Applic., 28, (1994), pp. 103-109.

[12] Y. Cherruault, G. Adomian, K. Abbaoui, and R. Rach, "Further remarks on convergence of decomposition method", International J. of Bio-Medical Computing., 38, (1995), pp. 89-93.

[13] K. Diethelm, and N. J. Ford, Numerical Solution of the Bagley-Torvik Equation, BIT 42, (2002), pp.490-507.

[14] A. Carpinteri, and F. Mainardi, Fractals and Fractional Calculus in Continuum Mechanics, Springer Verlag, Wien and New York 1997, pp. 223-276.

[15] G. F. D. Duff, and D. Naylor, Differential Equations of Applied Mathematics, Wiley \& Son, New York 1966. 